Excitatory synapses

\section{Is bigger better?}

Robert C. Malenka and Roger A. Nicoll

T he fundamental element of communication between neurons is the synapse, a subcellular specialization that requires the precise alignment of pre- and postsynaptic proteins. Over the past decade we have identified many of the molecular components involved in the exocytosis of neurotransmitter, but it has been more difficult to define the molecular architecture of postsynaptic sites, which differ in their protein composition depending on which neurotransmitter is used. A breakthrough in understanding excitatory synapses was the identification of a family of proteins that contain a PDZ domain. The properties of these proteins indicated that they may be important in the clustering and, perhaps, targeting of postsynaptic proteins ${ }^{1,2}$. Now, on page 433 of this issue, Migaud et al. ${ }^{3}$ describe a mouse with a targeted mutation in a prominent PDZ-domain protein, postsynaptic density-95 (PSD-95 $5^{4}$; also known as SAP9 $0^{5}$ ). Their findings confirm that PSD95 is a key component of excitatory synapses,

\section{Ocean chemistry}

\section{Iron and brimstone}

Spectacular layered sedimentary rocks, such as those pictured here from the Hamersley Basin of Western Australia, are a widespread yet episodic feature of the Precambrian geological landscape. These 'banded iron formations' - the colouring is largely due to variable iron content were laid down from an iron-rich ocean. Periods during which they accumulated are generally thought to have been ended by oxygenation of sub-surface ocean waters, forcing the oxidation and almost complete precipitation and deposition of dissolved iron from hitherto anoxic waters. However, as D. E. Canfield reports elsewhere in this issue (Nature 396, 450-453; 1998), such a deep-reaching breath of fresh air may not, after all, have been needed to relieve the ocean of its ferrous burden.

Canfield bases his case on a simple model of cycling of oceanic oxygen and nutrients, along with measured sulphurisotope signatures of sedimentary sulphide deposits and evidence of the emergence of various life forms. He argues that the ocean could have lost its iron from bottom waters that were akin to an oxygen-free sulphide 'soup'. Focusing on the Proterozoic Eon (from about 2.5 billion years ago to the end of the Precambrian around 540 million years ago), he proposes that known but also indicate a function other than that originally envisaged.

Excitatory synapses in the mammalian brain contain concentrated clusters of the NMDA ( $N$-methyl-D-aspartate) receptor, a subtype of glutamate receptor that is particularly important for triggering synaptic plasticity. Several years ago ${ }^{6}$, the cytoplasmic, carboxy-terminal tails of NMDAreceptor subunits were used as bait in a yeast two-hybrid screen and found to interact with PSD-95. This made perfect sense because PSD-95 was originally isolated ${ }^{4}$ as a prominent component of the postsynaptic density - the electron-dense thickening found at all excitatory synapses. We now know that PSD95 is one of a family of mammalian proteins that interact with NMDA-receptor subunits, as well as with certain potassium channels. This family also includes PSD-93/chapsyn110, SAP97/Hdlg and SAP-102, all of which contain three tandem PDZ domains at the amino terminus, an SH3 (src-homology) domain and a carboxy-terminal domain homologous to yeast guanylate kinase ${ }^{1,2}$. PDZ domains are thought to mediate protein-protein interactions and, in the case of the PSD-95 family, they bind to short amino-acid motifs at the carboxy termini of interacting proteins ${ }^{1,2}$.

Several lines of evidence support the idea that PSD-95 is involved in the synaptic clustering and targeting of NMDA receptors. First, both are highly concentrated at synapses ${ }^{6}$. Second, during development, PSD-95 clusters form opposite presynaptic terminals several days before synaptic NMDA clusters appear ${ }^{7}$. Third, when PSD95 or PSD-93/chapsyn-110 are coexpressed with NMDA receptors in heterologous cells (where these proteins are not normally expressed), clusters appear that do not form when the proteins are expressed alone ${ }^{8}$. Finally, mutations in the Drosophila homologue of PSD-95, Dlg, abolish the synaptic clustering of Shaker potassium channels ${ }^{9}$.

Although highly suggestive, all of this evidence is indirect, so neurobiologists have eagerly anticipated the first report of mice that lack PSD-95. This feat has now been accomplished by Migaud et al. ${ }^{3}$. They placed a stop codon in the third PDZ domain of PSD-95, leaving only the first two domains intact. The first surprising finding is that,

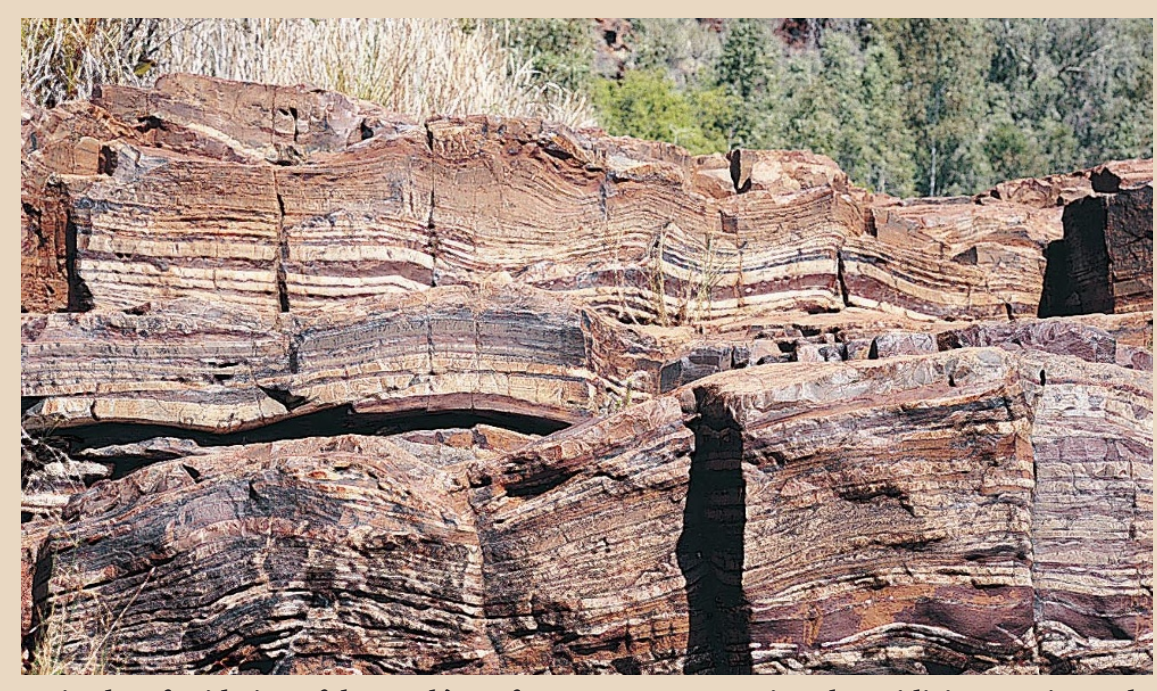

episodes of oxidation of the Earth's surface were insufficient to make the deep ocean aerobic until at least one billion years ago. If so, the end of the last great deposition of banded iron formations, some 1.8 billion years ago, stemmed not from the oxygenation of the deep - as generally held - but from some other process.

The sulphur-isotope data implicate a shift in the marine sulphur cycle as an alternative explanation. Canfield hypothesizes that a known oxidation of the Earth's surface about two billion years ago increased seawater sulphate concentrations by oxidizing continental mineral sulphides. Such an increase could have relieved existing limitations on the oceanic rate of sulphate reduction to sulphide, so allowing sufficient buildup of sulphide to precipitate out the ocean's dissolved iron as iron sulphides. On this analysis, the great oxygenation of the ocean interior did not occur for about another billion years, following another Earth-oxidation event which swept the deep waters clean of sulphide and, perhaps literally, breathed new life into the ocean. 
although PSD-95 is no longer found at synapses in the knockout mice, NMDA receptors are concentrated appropriately and generate normal synaptic responses. Thus, PSD-95 is not required for the synaptic clustering of NMDA receptors. Of course, other members of the PSD-95 family, such as PSD-93/chapsyn-110, may have served this role.

The second surprising finding comes from the study of synaptic plasticity in the CA1 region of the hippocampus. Normally, depending on the specific pattern of synaptic activation $^{10}$, CA1 synapses show both longterm potentiation (LTP) and long-term depression (LTD). In the absence of PSD-95, not only did Migaud and colleagues find that LTP was of much greater magnitude than normal, but the synaptic activity that normally elicits LTD now generated robust LTP. In other words, the absence of PSD-95 disrupted the rules by which synaptic activity is translated into long-lasting changes in synaptic strength. Presumably, this alteration explains why the mutant mice were considerably impaired in a hippocampaldependent learning task.

Why were these synaptic-plasticity mechanisms disrupted? As well as NMDA receptors ${ }^{6,8}$ PSD-95 can interact with other proteins, some of which are involved in signalling ${ }^{11}$. So, like other PDZ-domain proteins ${ }^{1,2,11,12}$, PSD-95 has been proposed to act as a scaffold that couples a signalling complex to activation of NMDA receptors. Presumably, without PSD-95 some component of the signalling complex that constrains LTP and triggers LTD has been lost. What might this be? One model for the bidirectional control of synaptic strength ${ }^{10}$ involves a tug-ofwar between protein kinase (LTP) and protein phosphatase activity (LTD), so perhaps a critical protein phosphatase in the post- synaptic density is missing. To date, however, there is no evidence for an interaction between phosphatases and PSD-95.

Another intriguing finding is that the PSD-95 mutant mice showed an increase in paired-pulse facilitation - a short-lived, activity-dependent increase in neurotransmitter release. This suggests that the lack of postsynaptic PSD-95 has influenced presynaptic function, perhaps by disrupting the interaction with other proteins (such as a recently identified presynaptic tripartite complex $^{13}$ ).

Migaud and colleagues ${ }^{3}$ elegantly show that we now have the tools to understand the molecular architecture of excitatory synapses in the mammalian brain. Their study also emphasizes the surprises that are in store as we try to work out how a specific protein embedded in this complex architecture leads to synaptic function and, eventually, to behaviour.

Robert C. Malenka is in the Departments of Psychiatry and Physiology, and Roger A. Nicoll is in the Departments of Cellular and Molecular

Physiology and Physiology, University of California, San Francisco, California 94143, USA.

e-mails:malenka@itsa.ucsf.edu

\section{nicoll@phy.ucsf.edu}

$$
\begin{aligned}
& \text { 1. Sheng, M. Neuron 17, 575-578 (1996). } \\
& \text { 2. Kornau, H.-C., Seeburg, P. H. \& Kennedy, M. B. Curr. Opin. } \\
& \text { Neurobiol. 7, 368-373 (1997). } \\
& \text { 3. Migaud, M. et al. Nature 396, 433-439 (1998). } \\
& \text { 4. Cho, K.-H., Hunt, C. A. \& Kennedy, M. B. Neuron 9, 929-942 } \\
& \text { (1992). } \\
& \text { 5. Kistner, V. et al. J. Biol. Chem. 268, 4580-4583 (1993). } \\
& \text { 6. Kornau, H.-C., Schenker, L. T., Kennedy, M. B. \& Seeburg, P. H. } \\
& \text { Science 269, 1737-1740 (1995). } \\
& \text { 7. Rao, A., Kim, E., Sheng, M. \& Craig, A. M. J. Neurosci. 18, } \\
& \text { 1217-1229 (1998). } \\
& \text { 8. Kim, E., Cho, K.-O., Rothschild, A. \& Sheng, M. Neuron 17, } \\
& \text { 103-113 (1996). } \\
& \text { 9. Tejedor, F. J. et al. J. Neurosci. 17, 152-159 (1997). } \\
& \text { 10. Malenka, R. C. Cell 78, 535-538 (1994). } \\
& \text { 11. Craven, S. E. \& Bredt, D. S. Cell 93, 495-498 (1998). } \\
& \text { 12. Tsunoda, S. et al. Nature 388, 243-249 (1997). } \\
& \text { 13. Butz, S., Okamoto, M. \& Südhof, T. C. Cell 94, 773-782 (1998). }
\end{aligned}
$$

Adding an extra air hole, or removing a hole, creates a core through which light is guided. Knight et al. ${ }^{5}$ previously made a photonic crystal fibre with a missing air hole at its centre to form a solid core. This fibre has some remarkable properties - however large the core, it supports only one optical mode (an intensity distribution of light that remains constant as it travels along the fibre $)^{6}$. This type of fibre would also transmit very high optical powers in comparison to conventional fibres, not because the mode is in air but because the area containing the

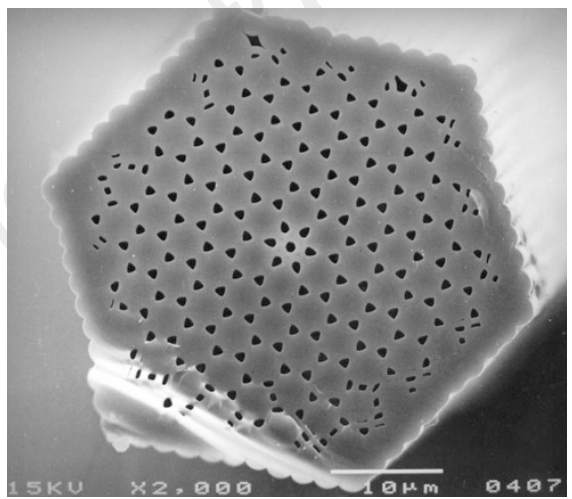

Figure 1 Cross-section of the photonic crystal fibre of Knight et al. ${ }^{1}$. The fibre is made by stacking a few hundred solid silica rods and hollow capillary tubes (which create the air holes) in a close-packed hexagonal pattern to make a preform, which is then drawn on a fibrepulling tower at a temperature of about $2,000{ }^{\circ} \mathrm{C}$. The lateral dimensions shrink by a factor of around 10,000, while becoming extended in the third direction. The holes run through the length of the fibre.

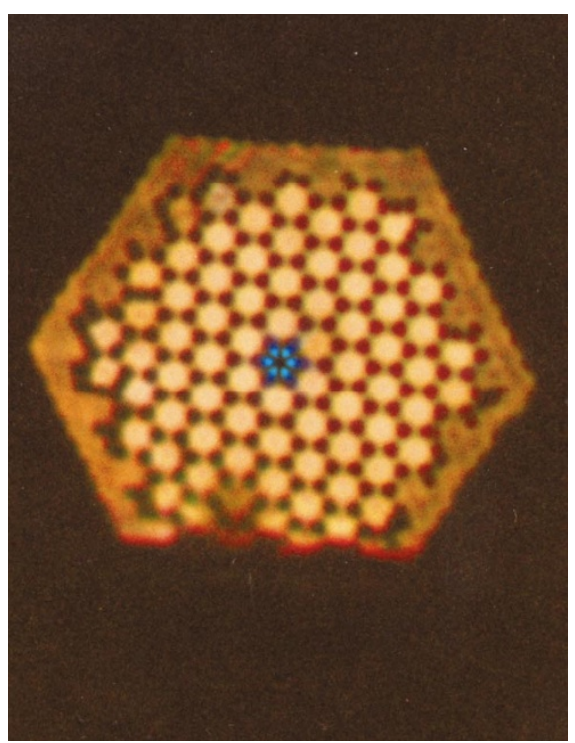

Figure 2 Guided light. In the photonic crystal fibre, the shape of the guided mode (blue) is influenced by six small holes about the main core (see Fig. 1). These survive near the centre of the fibre, but have collapsed elsewhere because of the forces introduced by the central hole. lattice (Fig. 1). This fibre is an example of a photonic crystal - a structure that repeats in one or more directions in space $\mathrm{e}^{2,3}$. As a result of multiple reflections, certain wavelengths cannot propagate in these structures. A structure is said to possess a 'photonic bandgap' if it reflects a wavelength incident from any angle in space.

In the early days of this field, it seemed that a large contrast in refractive index, such as that between air and a semiconductor (which has a refractive index, $n$, greater than 3 ), would be needed to achieve a photonic bandgap. But Birks et al. ${ }^{4}$ showed in theory that it is possible to create a two-dimensional photonic bandgap using the modest contrast in refractive index between air and silica $(n=1.5)$, providing that the light has a component travelling parallel to the direction of the rods. 